\title{
ANIMAL BREEDING AND THE ESTIMATION OF GENETIC VALUE
}

\author{
R. JARDINE \\ Department of Agriculture, Melbourne. Australia
}

Received 26.ii. 58

\section{INTRODUCTION}

IN the field of animal breeding, the determination of optimum selection methods requires that the genetic value $(Y)$ of an individual $(A)$ be estimable from such data as- $A$ 's phenotypic value $(y)$, the mean phenotypic values of $A$ 's $n$ full sibs $(F) ; A$ 's $m \bar{n}$ half sibs $(\bar{H})$, comprising $m$ families of full sibs, the $i^{\text {th }}$ with $n_{i}$ members, $\bar{n}=\left(\stackrel{m}{\sum n_{i}}\right) / m ; A$ 's $p \bar{q}$ progeny $(\bar{P})$, comprising $p$ families of full sibs, the $i^{\text {th }}$ with $q_{i}$ members, $\bar{q}=\left(\stackrel{p}{\sum} q_{i}\right) / p ;$ and such combinations of these as $\bar{F}^{\prime}=(y+n F) /(\mathrm{I}+n)$, $\bar{S}=(n \bar{F}+m \bar{n} \bar{H}) /(n+m \bar{n})$ and $\bar{S}^{\prime}=(y+n \bar{F}+m \bar{n} \bar{H}) /(1+n+m \bar{n})$. This estimate is obtainable on the assumption of a suitable genetic model and an application of the statistical technique of multiple regression to $Y$ and the subset of the means $y, \bar{F}, \bar{H}, \bar{P}, \bar{F}^{\prime}, \bar{S}, \bar{S}^{\prime}$ on which it is to be based.

As is evident from the work of Lush (1945), Lerner (1950) and Osborne (1957), the genetic model almost invariably adopted is one in which the phenotypic value of an individual is considered as due to independent additive factors with superimposed non-interacting random and non-random environmental effects, and, although more sophisticated formulations have been developed, this simple model appears to be adequate in many situations and is, in practice, the basis of most agricultural advice as to optimum breeding plans.

However, there has been no systematic treatment of the various possible cases of interest and, although most of the simple regression formulae have been presented at one time or another, those of multiple regression have been generally neglected, as witness Lerner et al. (I947) requiring the partial regression coefficients of $r$ on $y, F$ and $\bar{P}$ and of $Y$ on $\vec{F}$ and $P$ and applying instead the simple regression coefficients. In fact, the only case of multiple regression fully discussed has been that of the estimation of $Y$ from $y$ and $F$, Lush (1947), and although this has been extended by Osborne (1957) to the estimation of $Y$ from $y, F$ and $\vec{H}$ the discussion is subject to the restrictions that half sib families are all of equal size (i.e. $n_{i}=\bar{n}$ for all $i$ ) and nonrandom environmental effects are absent.

In the following sections, therefore, the various cases of estimation are considered systematically and explicit formulæ for the regression coefficients and the relative selective advance are derived, arbitrary 
numbers of relatives and non-random environmental effects being allowed. The necessary modifications when all individuals are inbred are also indicated.

\section{GENETIC MODEL}

Each individual involved in the estimation is considered to be a member of a hypothetical population in which phenotypic value, $y$, is given by-

$$
y=M+g_{1}+g^{\prime}{ }_{1}+g_{2}+g^{\prime}{ }_{2}+\ldots+g_{k}+g^{\prime}{ }_{k}+u+c+e,
$$

the joint variations of these individuals being subject only to restrictions consequent on genetic relationship and common environment. $M$ is a constant and the other symbols random variables, $g_{i}$ and $g_{i}{ }_{i}$ describing the effects of the two genes representing the $i^{\text {th }}$ factor, $u$ and $c$ nonrandom and $e$ a random environmental effect. The variables $g_{i}$ and $g^{\prime}{ }_{i}$ may assume values $a_{i}$ or $b_{i}$ with probabilities $p_{i}$ and $q_{i}$, respectively, where $p_{i}+q_{i}=\mathrm{I}$ and $p_{i} a_{i}+q_{i} b_{i}=0$. The non-random environmental effects are due to individuals sharing a common environment, $u$ being assumed to take the same value for full sibs and $c$ the same value for all individuals housed together, and either or both may be assumed zero as appropriate. In the cases considered below, $c$ takes the same value for all individuals full or half sib to each other and of the same generation. Genetic value, $Y$, is given by-

$$
r=M+g_{1}+g^{\prime}{ }_{1}+g_{2}+g^{\prime}{ }_{2}+\ldots+g_{k}+g^{\prime}{ }_{k}
$$

and all random variables in the model are of mean zero and uncorrelated (except as below) in accordance with the assumptions previously adopted.

Using the term locus to refer to a specific section of a chromosome, two loci may be defined as identical if they have both been derived from some one locus at a previous meiotic division, so that, excepting mutation, the same allele of a factor is represented by the genes at those loci. Then, following Malécot (1948), one may define the coefficient of inbreeding (c.i.), $f$, of an individual as the probability that the two loci representing any factor are identical, and the coefficient of relationship (c.r.), $g$, of two individuals as the probability that two loci representing any factor, one being nominated at random from each individual, are likewise identical. Thus, the coefficient of inbreeding of an individual is the coefficient of relationship of its parents, and the population to which the probabilities refer is hypothetical and generated by infinite repetitions of the pattern of matings antecedent to the individuals being considered. For non-inbred individuals, the c.r. of an individual $A$ with itself is $\frac{1}{2}$; of $A$ and $A$ 's full sib $\frac{1}{4}$; of $A$ and $A$ 's progeny $\frac{1}{4}$; of $A$ and $A$ 's half sib $\frac{1}{8}$; of $A$ 's full sib and $A$ 's progeny $\frac{1}{8}$; and of $A$ 's half sib and $A$ 's progeny $\frac{1}{16}$.

Then, if an individual has a c.i. $f$, expected values are such that $E\left(g_{i} g^{\prime}{ }_{i}\right)=f . E\left(g_{i}\right)^{2}=f . E\left(g^{\prime}{ }_{i}\right)^{2}$, and, similarly, if the c.r. of two 
individuals is $g$ (barred symbols indicating the second individual),

$$
E\left(g_{i} \bar{g}_{i}\right)=E\left(g_{i} \bar{g}_{i}^{\prime}\right)=E\left(g_{i}^{\prime} \bar{g}_{i}\right)=E\left(g_{i}^{\prime} \bar{g}_{i}^{\prime}\right)=g . E\left(g_{i}\right)^{2}=g . E\left(\bar{g}_{i}\right)^{2} .
$$

Define $V_{G}={ }^{k} \Sigma E\left(g_{i}\right)^{2}, \quad V_{U}=E(u)^{2}, \quad V_{C}=E(c)^{2}, \quad V_{E}=E(e)^{2}, \quad V=$ $V_{G}+V_{U}+V_{C}+V_{E}$ and $h^{2}=V_{G} / V, u^{2}=V_{U} / V, c^{2}=V_{C} / V, e^{2}=V_{E} / V$, so that $h^{2}+u^{2}+c^{2}+e^{2}=\mathrm{I}$. The heritability of the character being considered is $h^{2}$ and the reproductibility $\left(h^{2}+u^{2}+c^{2}\right)$.

\section{ESTIMATION FORMULAE}

The genetic value $r$ of an individual is to be estimated from the values of some selection of the means $y, F, \bar{H}, \bar{P}, F^{\prime}, \bar{S}$ and $\bar{S}^{\prime}$, and the appropriate formulae are listed below for a variety of cases, being set out as :-

(a) The means on which the estimate is to be based.

(b) The corresponding simple or partial regression coefficients.

(c) The variance of $Y$ accounted for by the introduction of successive means in the order indicated. The ielative

- selective advance is the square root of the sum of these items divided by $h^{2}$.

These formulae provide, in effect, average values over a population defined by the partition of the variance of phenotypic value as $h^{2}+u^{2}+c^{2}+e^{2}=\mathrm{I}$. Define $T=\left(\frac{1}{2} h^{2}+u^{2}+c^{2}\right), \quad T=\left(\frac{1}{4} h^{2}+c^{2}\right)$, $k=\left(\sum_{,}^{m} n_{i}{ }_{i}\right) / m \bar{n}^{2}$ and $l=\left(\sum_{,}^{p} q_{i}^{2}\right) / p \bar{q}^{2}$. Then-

I. (a) $y$.

(b) $b_{y} y, h^{2}$

(c) $y, \quad h^{4}$

2. (a) $F$.
(b) $b_{Y \bar{F}}, \frac{n h^{2}}{2[\mathrm{I}+(n-\mathrm{I}) T]}$
(c) $F, \frac{n h^{4}}{4[\mathrm{I}+(n-\mathrm{I}) T]}$

3. (a) $A$.
(b) $b_{Y \bar{H}}, \frac{m \bar{n} h^{2}}{4[\mathrm{I}+(k \bar{n}-\mathrm{I}) T+\bar{n}(m-k) \bar{T}]}$

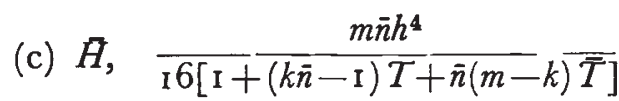


4. (a) $\bar{P}$.

(b) $b_{Y \bar{P}}, \frac{p \bar{q} h^{2}}{2[\mathrm{I}+(l \bar{q}-\mathrm{I}) T+\bar{q}(p-l) \bar{T}]}$

(c) $\bar{P}, \frac{p \bar{q} h^{4}}{4[\mathrm{I}+(l \bar{q}-\mathrm{I}) \mathcal{T}+\bar{q}(p-l) \bar{T}]}$

5. (a) $F^{\prime}$.

(b) $b_{Y \bar{F}^{\prime}}, \frac{(n+2) h^{2}}{2(I+n T)}$

(c) $F^{\prime}, \frac{(n+2)^{2} h^{4}}{4(n+\mathrm{I})(\mathrm{I}+n T)}$

6. (a) $\bar{S}$.

(b) Define $m^{\prime}=(m+\mathrm{I}), \bar{n}^{\prime}=(n+m \tilde{n}) /(m+\mathrm{I})$, $k^{\prime}=(m+\mathrm{I})\left(n^{2}+m \bar{n}^{2} k\right) /(n+m \bar{n})^{2}$

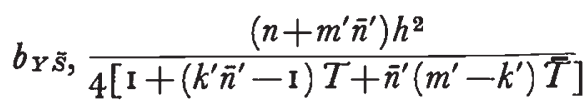

(c) $\bar{S}, \frac{\left(n+m^{\prime} \bar{n}^{\prime}\right)^{2} h^{4}}{\mathrm{I} 6 m^{\prime} \bar{n}^{\prime}\left[\mathrm{I}+\left(k^{\prime} \bar{n}^{\prime}-\mathrm{I}\right) T+\bar{n}^{\prime}\left(m^{\prime}-k^{\prime}\right) \bar{T}\right]}$

7. (a) $\bar{S}^{\prime}$.

(b) Define $m^{\prime}=(m+\mathrm{I}), \bar{n}^{\prime}=(\mathrm{I}+n+m \bar{n}) /(m+\mathrm{I})$, $k^{\prime}=(m+\mathbf{I})\left[(n+\mathbf{I})^{2}+m \bar{n}^{2} k\right] /(\mathbf{I}+n+m \bar{n})^{2}$ $b_{Y \bar{S}^{\prime}}, \frac{\left(3+n+m^{\prime} \bar{n}^{\prime}\right) h^{2}}{4\left[\mathrm{I}+\left(k^{\prime} \bar{n}^{\prime}-\mathrm{I}\right) T+\bar{n}^{\prime}\left(m^{\prime}-k^{\prime}\right) \bar{T}\right]}$

(c) $\bar{S}^{\prime}, \frac{\left(3+n+m^{\prime} \bar{n}^{\prime}\right)^{2} h^{4}}{\mathrm{I} 6 m^{\prime} \bar{n}^{\prime}\left[\mathrm{I}+\left(k^{\prime} \bar{n}^{\prime}-\mathrm{I}\right) T+\bar{n}^{\prime}\left(m^{\prime}-k^{\prime}\right) \bar{T}\right]}$

8. (a) $y, F$.

(b) $b_{Y F} y, \frac{n(\mathrm{I}-2 T) h^{2}}{2(\mathrm{I}-T)(\mathrm{I}+n T)}$ $b_{Y} y \cdot \bar{F}, h^{2}-T b_{Y F} \cdot y$

(c) $y, \quad h^{4}$

$F, \quad \frac{n(\mathrm{I}-2 T)^{2} h^{4}}{4(\mathrm{I}-T)(\mathrm{I}+n T)}$

9. (a) $y, H$.

(b) $b_{Y \bar{H}} . y, \frac{m \bar{n}(\mathrm{I}-4 \bar{T}) h^{2}}{4\left[\mathrm{I}+(k \bar{n}-\mathrm{I}) \bar{T}+\bar{n}(m-k) \bar{T}-m \bar{n} \bar{T}^{2}\right]}$

$b_{Y} y \cdot \vec{H}, h^{2}-\bar{T} b_{Y \tilde{H}} \cdot y$

(c) $y, \quad h^{4}$

$H, \quad \frac{m \bar{n}(\mathrm{I}-4 \bar{T})^{2} h^{4}}{\mathrm{I} 6\left[\mathrm{I}+(k \bar{n}-\mathrm{I}) T+\bar{n}(m-k) \bar{T}-m \bar{n} \bar{T}^{2}\right]}$ 
xo. (a) $y, \bar{P}$.
(b) $b_{Y \bar{P}}, y, \frac{2 p \bar{q}\left(\mathrm{I}-h^{2}\right) h^{2}}{\left\{4[\mathrm{I}+(l \bar{q}-\mathrm{I}) T+\bar{q}(p-l) \bar{T}]-p \bar{q} h^{4}\right\}}$
$b_{Y} y_{. \tilde{P}},\left(\mathrm{I}-\frac{1}{2} b_{Y \bar{P}} \cdot y\right) h^{2}$
(c) $y, \quad h^{4}$
$\bar{P} \quad \frac{p \bar{q}\left(\mathrm{I}-h^{2}\right)^{2} h^{4}}{\left\{4[\mathrm{I}+(l \bar{q}-\mathrm{I}) T+\bar{q}(p-l) \bar{T}]-p \bar{q} h^{4}\right\}}$

I I. (a) $F, \bar{P}$.
(b) $b_{Y \bar{P}, \bar{F}}, \frac{2 p \bar{q}\left\{4[\mathrm{I}+(n-\mathrm{I}) T]-n h^{2}\right\} h^{2}}{\left\{\mathrm{I} 6[\mathrm{I}+(n-\mathrm{I}) T][\mathrm{I}+(l \bar{q}-\mathrm{I}) T+\bar{q}(p-l) \bar{T}]-n p \bar{q} h^{4}\right\}}$
$b_{Y \bar{F}, \bar{P}}, \frac{n}{2[\mathrm{I}+(n-\mathrm{I}) T]}\left(\mathrm{I}-\frac{1}{2} b_{Y \bar{P}, \bar{F}}\right) h^{2}$
(c) $F, \frac{n h^{\mathbf{4}}}{4[\mathrm{I}+(n-\mathrm{I}) T]}$
$\bar{P}, \frac{p \bar{q}\left\{4[\mathrm{I}+(n-\mathrm{I}) T]-n h^{2}\right\}^{2} h^{4}}{4[\mathrm{I}+(n-\mathrm{I}) T]\{\mathrm{I} 6[\mathrm{I}+(n-\mathrm{I}) T]}$

I2. (a) $F, \bar{H}$.

(b) $b_{Y \bar{H} . \bar{F}}, \frac{m \bar{n}[\mathrm{I}+(n-\mathrm{I}) T-2 n T] h^{2}}{4\left\{[\mathrm{I}+(n-\mathrm{I}) T][\mathrm{I}+(k \bar{n}-\mathrm{I}) T+\bar{n}(m-k) \bar{T}]-m \bar{n} n \bar{T}^{2}\right\}}$

$b_{Y \bar{F}, \bar{H}}, \frac{n}{2[\mathrm{I}+(n-\mathrm{I}) \bar{T}]}\left(h^{2}-2 \overline{\mathcal{T}} b_{Y \bar{H} \cdot \bar{F}}\right)$

(c) $F, \frac{n h^{4}}{4[\mathrm{I}+(n-\mathrm{I}) T]}$

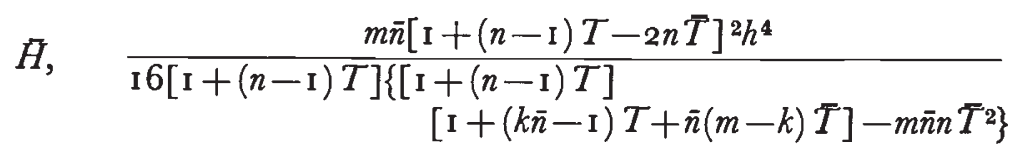

I3. (a) $\bar{H}, \bar{P}$. (where $n_{i}=\mathrm{I}$ for all $\left.i\right)$.

(b) $b_{Y \bar{P} . \bar{H}}, \frac{2 p \bar{q}\left\{\mathrm{I} 6[\mathrm{I}+(m-\mathrm{I}) T]-m h^{2}\right\} h^{2}}{\left\{64[\mathrm{I}+(m-\mathrm{I}) \bar{T}][\mathrm{I}+(l \bar{q}-\mathrm{I}) T+\bar{q}(p-l) \bar{T}]-m p \bar{q} h^{4}\right\}}$

$b_{Y \tilde{H} . \mathcal{P}}, \frac{m}{4[\mathrm{I}+(m-\mathrm{I}) \bar{T}]}\left(\mathrm{I}-\frac{1}{2} b_{Y \bar{P} \cdot \bar{H}}\right) h^{2}$

(c) $H, \frac{m h^{4}}{\mathrm{I} 6[\mathrm{I}+(m-\mathrm{I}) \bar{T}]}$

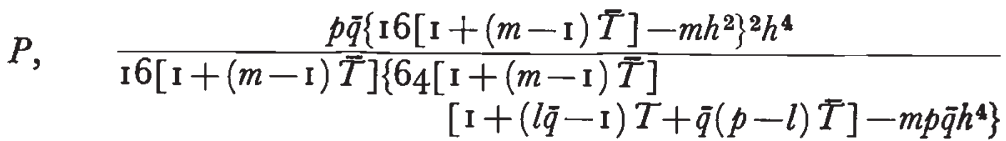


14. (a) $y, F, \bar{H}$.

(b) $b_{Y \bar{H}} \cdot y_{\bar{F}}, \frac{m \bar{n}[\mathrm{I}+n T-2(n+2) \bar{T}] h^{2}}{4\left\{(\mathrm{I}+n T)[\mathrm{I}+(k \bar{n}-\mathrm{I}) T+\bar{n}(m-k) \bar{T}]-m \bar{n}(n+\mathrm{I}) \bar{T}^{2}\right\}}$

$b_{Y \bar{F}} y_{\bar{H}}, \frac{n(\mathrm{I}-2 T) h^{2}}{2(\mathrm{I}-T)(\mathrm{I}+n T)}-\frac{n \bar{T}}{(\mathrm{I}+n T)} b_{Y \bar{H} \cdot y_{\bar{F}}}$

$b_{Y} y_{\bar{F} \bar{H}},\left(h^{2}-T b_{Y \bar{F}} \cdot y_{\bar{H}}-\bar{T} b_{Y \bar{H}} \cdot y_{\bar{F}}\right)$

(c) $y, \quad h^{4}$

$F, \quad \frac{n(\mathrm{I}-2 T)^{2} h^{4}}{4(\mathrm{I}-T)(\mathrm{I}+n T)}$

$H, \quad \frac{m \bar{n}[\mathrm{I}+n T-2(n+2) \bar{T}]^{2} h^{4}}{\mathrm{I} 6(\mathrm{I}+n T)\{(\mathrm{I}+n T)[\mathrm{I}+(k \bar{n}-\mathrm{I}) T}$

$\left.+\bar{n}(m-k) \bar{T}]-m \bar{n}(n+\mathrm{I}) \bar{T}^{2}\right\}$

This case is considered by Osborne (I957), and substituting (n-r) for $n,(d-\mathrm{I})$ for $m, n$ for $\bar{n}$ and I for $k$,

$$
\begin{aligned}
& b_{Y \vec{H}}, y_{\bar{F}}=\frac{n(d-\mathrm{I})[\mathrm{I}+(n-\mathrm{I}) T-2(n+\mathrm{I}) \bar{T}] h^{2}}{4[\mathrm{I}+(n-\mathrm{I}) T-n \tilde{T}][\mathrm{I}+(n-\mathrm{I}) T+n(d-\mathrm{I}) \bar{T}]} \\
& b_{Y \bar{F}} y_{\bar{H}}=\frac{(n-\mathrm{I})(\mathrm{I}-2 T) h^{2}}{2(\mathrm{I}-T)[\mathrm{I}+(n-\mathrm{I}) T]}-\frac{(n-\mathrm{I}) T}{[\mathrm{I}+(n-\mathrm{I}) T]} b_{Y \bar{H}} \cdot y_{\bar{F}}
\end{aligned}
$$

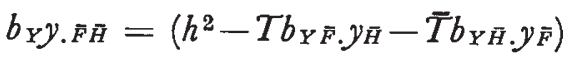

and Osborne's coefficients $b_{2}$ and $b_{3}$ are then given by-

$$
\begin{aligned}
& b_{2}=\frac{n(d-\mathrm{I}) b_{Y \bar{F}} \cdot y_{\bar{H}}-(n-\mathrm{I}) b_{Y \bar{H}} \cdot y_{\bar{F}}}{(d-\mathrm{I})\left[(n-\mathrm{I}) b_{Y} y_{\bar{F} \bar{H}}-b_{Y \bar{F}} \cdot y_{\bar{H}}\right]} \\
& b_{3}=\frac{d(n-\mathrm{I}) b_{Y \bar{H}} \cdot y_{\bar{F}}}{(d-\mathrm{I})\left[(n-\mathrm{I}) b_{Y} y_{\bar{F} \bar{H}}-b_{\left.Y \bar{F} \cdot y_{\bar{H}}\right]}\right.}
\end{aligned}
$$

I5. (a) $y, F, \bar{P}$.

(b) $\begin{aligned} b_{Y \bar{P}}, y \bar{F}, \frac{2 p \bar{q}\left\{4(\mathrm{I}-T)(\mathrm{I}+n T)-(4+n-4 T) h^{2}\right\} h^{2}}{\{\mathrm{I} 6(\mathrm{I}-T)(\mathrm{I}+n \bar{T})[\mathrm{I}+(l \bar{q}-\mathrm{I}) T+} \\ \left.\quad+\bar{q}(p-l) \bar{T}]-p \bar{q}(4+n-4 T) h^{4}\right\}\end{aligned}$

$b_{Y F}, y_{\bar{H}}, \frac{n(\mathrm{I}-2 T)}{2(\mathrm{I}-T)(\mathrm{I}+n T)}\left(\mathrm{I}-\frac{1}{2} b_{Y \tilde{P}} . y_{\bar{F}}\right) h^{2}$

$b_{Y} y_{\bar{F}} \bar{H},\left(h^{2}-T b_{Y \bar{F}} y_{\bar{H}}-\frac{1}{2} h^{2} b_{Y \bar{P}} \cdot y_{\bar{F}}\right)$

(c) $y, \quad h^{4}$

$F, \quad \frac{n(\mathrm{I}-2 T)^{2} h^{4}}{4(\mathrm{I}-T)(\mathrm{I}+n T)}$

$P, \quad \frac{p \bar{q}\left\{4(\mathrm{I}-T)(\mathrm{I}+n T)-(4+n-4 T) h^{2}\right\}^{2} h^{4}}{4(\mathrm{I}-T)(\mathrm{I}+n T)\{\mathrm{I} 6(\mathrm{I}-T)(\mathrm{I}+n T)[\mathrm{I}+(l \bar{q}-\mathrm{I}) T}$

$\left.+\bar{q}(p-l) \bar{T}]-p \bar{q}(4+n-4 T) h^{4}\right\}$ 
I6. (a) $y, \bar{H}, \bar{P}$ (where $n_{i}=$ I for all $i$ )

$$
\begin{aligned}
& \text { (b) } b_{Y \bar{P}} \cdot y_{\bar{H}}, \frac{2 p \bar{q}\left\{\mathrm{I} 6(\mathrm{I}-\bar{T})(\mathrm{I}+m \bar{T})-[\mathrm{I} 6+m+8(m-2) \bar{T}] h^{2}\right\} h^{2}}{\{64(\mathrm{I}-\bar{T})(\mathrm{I}+m \bar{T})[\mathrm{I}+(l \bar{q}-\mathrm{I}) \bar{T}+\bar{q}(p-l) \bar{T}]} \\
& \left.-p \bar{q}[\mathrm{I} 6+m+8(m-2) \bar{T}] h^{4}\right\} \\
& b_{Y \bar{H}} \cdot y_{\bar{P}}, \frac{m(\mathrm{I}-4 \bar{T})}{4(\mathrm{I}-\bar{T})(\mathrm{I}+m \bar{T})}\left(\mathrm{I}-\frac{1}{2} b_{Y \bar{P}} \cdot y_{\bar{H}}\right) h^{2} \\
& b_{Y y \cdot \bar{H} \bar{P}},\left(h^{2}-\bar{T} b_{Y \bar{H}} \cdot y_{\bar{F}}-\frac{1}{2} h^{2} b_{Y \bar{P}} \cdot y_{\bar{H}}\right) \\
& \text { (c) } y, \quad h^{4} \\
& H, \quad \frac{m(\mathrm{r}-4 \bar{T})^{2} h^{4}}{\mathrm{I} 6(\mathrm{I}-\bar{T})(\mathrm{I}+m \bar{T})} \\
& \bar{P}, \quad \frac{p \bar{q}\left\{\mathrm{I} 6(\mathrm{I}-\bar{T})(\mathrm{I}+m \bar{T})-[\mathrm{r} 6+m+8(m-2) \bar{T}] h^{2}\right\}^{2} h^{4}}{\mathrm{I} 6(\mathrm{I}-\bar{T})(\mathrm{I}+m \bar{T})\{64(\mathrm{I}-\bar{T})(\mathrm{I}+m \bar{T})[\mathrm{I}+(l \bar{q}-\mathrm{I}) T} \\
& \left.+\bar{q}(p-l) \bar{T}]-p \bar{q}[\mathrm{I} 6+m+8(m-2) \bar{T}] h^{4}\right\}
\end{aligned}
$$

\section{INBREEDING}

The formulae of the preceding section can all be generalised so as to apply to inbred individuals, the necessary coefficients of inbreeding and relationship being readily calculable, given the individuals on which the estimation is to be based and the pattern of matings which gave rise to them.

Consider, as an example, the random mating of a finite set of $M$ males and $F$ females where the coefficients of inbreeding and relationship in successive generations are $f^{\prime}, g^{\prime}$ and $f, g$, respectively. Then, if $H$ is the harmonic mean of $M$ and $F$, i.e. $2 M F /(M+F)$,

$$
f=g^{\prime}, g=\frac{\mathrm{I}}{H}\left[\frac{1}{4}+\frac{1}{4} f^{\prime}+\frac{1}{2}(2 H-\mathrm{I}) g^{\prime}\right],
$$

and the coefficient of relationship between individuals of the male (female) parent and progeny groups, $g_{M}\left(g_{F}\right)$, is given by substituting $M(F)$ for $H$ in the above formula for $g$. This recurrence relation is also applicable to other mating systems if appropriate values are given to $M$ and $F$. Thus for selfing $M=F=\frac{1}{2}, H=\frac{1}{2}$; for full sib mating $M=F=\mathrm{I}, H=\mathrm{I}$; for half sib mating $M=\mathrm{I}, F=\infty$, $H=2$; and for random mating (infinite population) $M=F=\infty$, $H=\infty$. Such coefficients may be calculated successively from an initial stage when individuals are non-inbred and unrelated, and are applicable in such formulæ as are given in the next section.

Moreover, when pedigrees are known precisely, it may be noted that, while the coefficients of inbreeding and relationship of specific individuals refer (as previously stated) to the probability of certain loci being identical, it is, of course, a matter of chance, subject to such probabilities, whether those loci are or are not actually identical. This latter statement is equally true for the random mating considered 
above, where pedigrees are not precisely known (any two individuals may actually have $\mathrm{o}$, I or 2 parents in common), but now the coefficients do not give the probability of certain loci being identical for the specific individuals concerned, but the average of such values as are generated by the random choice of parents for those individuals.

\section{ESTIMATION FORMULAE-INDIVIDUALS INBRED}

Consider an individual $A$ and the groups of relatives specified in section I. In $A$ 's generation the c.i. and c.r. within full sib groups are $f^{\prime}$ and $g^{\prime}$, respectively, and the c.r. between full sib groups, $\bar{g}^{\prime}$. Similarly, in the progeny generation the c.i. and c.r. within full sib groups are $f$ and $g$, respectively, and the c.r. between full sib groups, $\bar{g}$. In addition, the c.r. between $A$ 's progeny and $A$ is $G ; A$ 's progeny and $A$ 's full sibs, $\bar{G}$; and $A$ 's progeny and $A$ 's half sibs, $\bar{G}^{\prime}$.

Define $S^{\prime}=\left(\mathrm{I}+f^{\prime} h^{2}\right), S=\left(\mathrm{I}+f h^{2}\right), T^{\prime}=\left(2 g^{\prime} h^{2}+u^{2}+c^{2}\right), \quad T=$ $\left(2 g h^{2}+u^{2}+c^{2}\right), \bar{T}^{\prime}=\left(2 \bar{g}^{\prime} h^{2}+c^{2}\right), \bar{T}=\left(2 \bar{g} h^{2}+c^{2}\right)$ and all other symbols as before. When individuals are non-inbred, $f^{\prime}=0, g^{\prime}=\frac{1}{4}, \bar{g}^{\prime}=\frac{1}{8}$, $f=0, g=\frac{1}{4}, \bar{g}=\frac{1}{8}, G=\frac{1}{4}, \bar{G}=\frac{1}{8}, \bar{G}^{\prime}=\frac{1}{16}$, as previously stated, and $S^{\prime}=S=\mathrm{I}, \bar{T}^{\prime}=T, \bar{T}^{\prime}=\bar{T}$. The following variances and covariances for the general case may now be derived :-

$$
\begin{aligned}
& V(r)=\left(\mathrm{I}+f^{\prime}\right) h^{2} \\
& V(y)=S^{\prime} \\
& V(\bar{F})=\frac{S^{\prime}+(n-\mathrm{I}) T^{\prime}}{n} \\
& V(\bar{H})=\frac{S^{\prime}+(k \bar{n}-\mathrm{I}) T^{\prime}+\bar{n}(m-k) \bar{T}^{\prime}}{m \bar{n}} \\
& V(\bar{P})=\frac{S+(l \bar{q}-\mathrm{I}) T+\bar{q}(p-l) \bar{T}}{p \bar{q}} \\
& V\left(F^{\prime}\right)=\frac{S^{\prime}+n T^{\prime}}{(n+\mathrm{I})}
\end{aligned}
$$

$V(\bar{S}) \quad$ as for $V(\bar{H})$ substituting $m^{\prime}, \bar{n}^{\prime}, k^{\prime}$ for $m, \bar{n}, k$ where

$$
\begin{aligned}
m^{\prime} & =(m+\mathrm{I}), \bar{n}^{\prime}=\frac{(n+m \bar{n})}{(m+\mathrm{I})}, \\
k^{\prime} & =\frac{(m+\mathrm{I})\left(n^{2}+m \bar{n}^{2} k\right)}{(n+m \bar{n})^{2}}
\end{aligned}
$$

$V\left(\bar{S}^{\prime}\right) \quad$ as for $V(\bar{H})$ substituting $m^{\prime}, \bar{n}^{\prime}, k^{\prime}$ for $m, \bar{n}, k$ where

$$
\begin{aligned}
m^{\prime} & =(m+\mathrm{I}), \bar{n}^{\prime}=\frac{(\mathrm{I}+n+m \bar{n})}{(m+\mathrm{I})}, \\
k^{\prime} & =\frac{(m+\mathrm{I})\left[(n+\mathrm{I})^{2}+m \bar{n}^{2} k\right]}{(\mathrm{I}+n+m \bar{n})^{2}}
\end{aligned}
$$




$$
\begin{aligned}
& W(Y y)=\left(\mathrm{I}+f^{\prime}\right) h^{2} \\
& W(Y \bar{F})=2 g^{\prime} h^{2} \\
& W(Y \bar{H})=2 \bar{g}^{\prime} h^{2} \\
& W(Y \bar{P})=2 G h^{2} \\
& W\left(Y \bar{F}^{\prime}\right)=\frac{\left[\mathrm{I}+f^{\prime}+2 n g^{\prime}\right] h^{2}}{(n+\mathrm{I})} \\
& W(Y \bar{S})=\frac{2\left[n\left(g^{\prime}-\bar{g}^{\prime}\right)+m^{\prime} \bar{n}^{\prime} \bar{g}^{\prime}\right] h^{2}}{(n+m \bar{n})} \\
& W\left(Y \overline{S^{\prime}}\right)=\frac{\left[\mathrm{I}+\left(f^{\prime}-2 \bar{g}^{\prime}\right)+2 n\left(g^{\prime}-\bar{g}^{\prime}\right)+2 m^{\prime} \bar{n}^{\prime} \bar{g}^{\prime}\right] h^{2}}{(\mathrm{I}+n+m \bar{n})} \\
& W(y \bar{F})=T^{\prime} \\
& W(y \bar{H})=\bar{T}^{\prime} \\
& W(y \bar{P})=2 G h^{2} \\
& W(\bar{F} \bar{H})=\bar{T}^{\prime} \\
& W(\overline{F P})=2 \bar{G} h^{2} \\
& W(\overline{H P})=2 \dot{G}^{\prime} h^{2}
\end{aligned}
$$

The regression analysis of $X$ for any selection of the independent variables may now be carried through numerically, for any particular case, by the use of these formulae or, alternatively, the regression formulae calculated explicitly (as previously and when not inordinately long), numerical values being substituted later. Although these more general formulae do not appear to be particularly useful, a selection of the simpler cases is given below and set out as before. The relative selective advance is now the square root of the sum of the items listed under (c) divided by $\left(\mathrm{I}+f^{\prime}\right) h^{2} / \sqrt{ } S^{\prime}$, rather than $h^{2}$ as before.

I. (a) $y$
(b) $b_{Y y}, \frac{\left(\mathrm{I}+f^{\prime}\right) h^{2}}{S^{\prime}}$
(c) $y, \quad \frac{\left(\mathrm{I}+f^{\prime}\right)^{2} h^{4}}{S^{\prime}}$

2. (a) $F$

(b) $b_{Y \bar{F}}, \frac{2 n g^{\prime} h^{2}}{S^{\prime}+(n-\mathrm{I}) T^{\prime}}$

(c) $F, \frac{4 n\left(g^{\prime}\right)^{2} h^{4}}{S^{\prime}+(n-I) T^{\prime}}$ 
3. (a) $F^{\prime}$.
(b) $b_{Y \bar{F}^{\prime}}, \frac{\left(\mathrm{I}+f^{\prime}+2 n g^{\prime}\right) h^{2}}{S^{\prime}+n T^{\prime}}$
(c) $F^{\prime}, \frac{\left(\mathrm{I}+f^{\prime}+2 n g^{\prime}\right)^{2} h^{4}}{(n+\mathrm{I})\left(S^{\prime}+n T^{\prime}\right)}$

4. (a) $\bar{P}$.
(b) $b_{Y \bar{P}}, \frac{2 p \bar{q} G h^{2}}{[S+(l \bar{q}-\mathrm{I}) T+\bar{q}(p-l) \bar{T}]}$
(c) $P, \frac{4 p \bar{q} G^{2} h^{4}}{[S+(l \bar{q}-\mathrm{I}) T+\bar{q}(p-l) \bar{T}]}$

5. (a) $y, F$.

(b) $b_{Y \bar{F}} y, \frac{n\left[2 g^{\prime} S^{\prime}-\left(\mathrm{I}+f^{\prime}\right) T^{\prime}\right] h^{2}}{\left(S^{\prime}-T^{\prime}\right)\left(S^{\prime}+n T^{\prime}\right)}$

$$
b_{Y} y . \bar{F}, \frac{\left(\mathrm{I}+f^{\prime}\right) h^{2}}{S^{\prime}}-\frac{T^{\prime}}{S^{\prime}} b_{Y \bar{F}} \mathcal{Y}
$$

(c) $y, \quad \frac{\left(1+f^{\prime}\right)^{2} h^{4}}{S^{\prime}}$

$$
F, \frac{n\left[2 g^{\prime} S^{\prime}-\left(\mathrm{I}+f^{\prime}\right) T^{\prime}\right]^{2} h^{4}}{S^{\prime}\left(S^{\prime}-T^{\prime}\right)\left(S^{\prime}+n T^{\prime}\right)}
$$

6. (a) $y, P$. (where $p=\mathrm{I}, \bar{q}=q)$

$$
\begin{aligned}
& \text { (b) } b_{Y \bar{P}} . y, \frac{2 q G\left(1-h^{2}\right) h^{2}}{\left\{S^{\prime}[S+(q-1) T]-4 q G^{2} h^{4}\right\}} \\
& b_{Y \mathcal{y} . \bar{P},} \frac{\left(1+f^{\prime}\right) h^{2}}{S^{\prime}}-\frac{2 G h^{2}}{S^{\prime}} b_{Y \vec{P}} \cdot y \\
& \text { (c) } \mathcal{Y}, \frac{\left(1+f^{\prime}\right)^{2} h^{4}}{S^{\prime}} \\
& \bar{P}, \frac{4 q G^{2}\left(1-h^{2}\right)^{2} h^{4}}{S^{\prime}\left\{S^{\prime}[S+(q-1) T]-4 q G^{2} h^{4}\right\}}
\end{aligned}
$$

7. (a) $F, P$. (where $p=\mathrm{I}, \bar{q}=q)$

(b) $b_{Y \bar{P} . \bar{F}}, \frac{2 q\left\{G\left[S^{\prime}+(n-I) T^{\prime}\right]-2 n \bar{G} g^{\prime} h^{2}\right\} h^{2}}{\left\{\left[S^{\prime}+(n-I) T^{\prime}\right][S+(q-I) T]-4 n q \bar{G}^{2} h^{4}\right\}}$

$$
b_{Y \bar{F} \cdot \bar{P}}, \frac{2 n}{\left[S^{\prime}+(n-!) T^{\prime}\right]}\left(g^{\prime}-\bar{G} b_{Y \bar{P} \cdot \bar{F}}\right) h^{2}
$$

(c) $F, \frac{4 n\left(g^{\prime}\right)^{2} h^{4}}{\left[S^{\prime}+(n-\mathrm{I}) T^{\prime}\right]}$

$P, \quad \frac{4 q\left\{G\left[S^{\prime}+(n-I) T^{\prime}\right]-2 n \bar{G} g^{\prime} h^{2}\right\}^{2} h^{4}}{\left[S^{\prime}+(n-I) T^{\prime}\right]\left\{\left[S^{\prime}+(n-I) T^{\prime}\right][S+(q-I) T]-4 n q \bar{G}^{2} h^{4}\right\}}$ 


\section{DIFFERENCES IN GENETIC VALUE}

When related individuals are to be compared, the foregoing formula require modification, and several cases are discussed below.

\section{(i) Sib tests}

Consider two groups of individuals, $A$ and $A$ 's $m$ associates and $B$ and $B$ 's $n$ associates. The c.i. and c.r. within the groups are $f^{\prime}$ and $g^{\prime}$, respectively, and the c.r. between the groups is $\tilde{g}^{\prime}$. The fraction of phenotypic variance due to common environment within the group is $u^{2}$.

(a) $\left(Y_{A}-Y_{B}\right)$ is to be estimated from either $\left(y_{A}-y_{B}\right)$ or $\left(F_{A}-F_{B}\right)$ or both jointly. Defining $K=2 m n /(m+n)$, the regression formulae required are those given as $\mathrm{I}, 2$ and 5 of section 5 when $\left(f^{\prime}-2 \bar{g}^{\prime}\right)$ is substituted for $f^{\prime},\left(g^{\prime}-\bar{g}^{\prime}\right)$ for $g^{\prime}, K$ for $n$ and with $c^{2}=0$.

(b) $\left(Y_{A}-\Upsilon_{B}\right)$ is to be estimated from $\left(\bar{F}_{A}^{\prime}-F^{\prime}{ }_{B}\right)$. The regression formulae required are those given as 3 of section 5 where $K^{\prime}$ is substituted for $(n+\mathrm{I})\left[K^{\prime}\right.$ being $\left.2(m+\mathrm{I})(n+\mathrm{I}) /(m+n+2)\right]$, $\left(f^{\prime}-2 \bar{g}^{\prime}\right)$ and $\left(g^{\prime}-\bar{g}^{\prime}\right)$ replacing $f^{\prime}$ and $g^{\prime}$ as above and with $c^{2}=0$.

\section{(ii) Progeny tests}

Consider an individual $A$ together with $A$ 's $p$ progeny and an individual $B$ together with $B$ 's $q$ progeny. The c.i. and c.r. within the progeny groups are $f$ and $g$, respectively, and the c.r. between the groups is $\bar{g}$. In addition, the c.r. of $A$ and $A$ 's progeny (likewise, $B$ and $B$ 's progeny) is $G$, while the c.r. of $A$ and $B$ 's progeny (likewise, $B$ and $A$ 's progeny) is $G_{0}$. The fraction of phenotypic variance due to common environment within the groups is again $u^{2}$.

$\left(\Upsilon_{A}-\Upsilon_{B}\right)$ is to be estimated from $\left(\bar{P}_{A}-\bar{P}_{B}\right)$. Defining $L=$ $2 p q /(p+q)$, the regression formulae required are those given as 4 of section 5 (for the case where $p=\mathrm{I}, \bar{q}=q)$ when $(f-2 \bar{g})$ is substituted for $f,(g-\bar{g})$ for $g,\left(G-G_{0}\right)$ for $G, L$ for $q$ and with $c^{2}=0$.

\section{DISCUSSION}

The greatest scope for the use of formulae such as have been considered in the previous sections is in the field of poultry breeding and, although it is not intended to discuss alternative breeding plans here, several points mentioned in the introduction, and referring to this application, may be considered briefly.

Firstly, as a matter of interest, the use of simple instead of partial regression coefficients may be examined, for Lerner's example and numerical values are given in table $\mathrm{I}$. The actual gain was $5^{\circ} 6$ eggs, the estimate using simple regression coefficients 5.28 eggs, and that using partial regression coefficients 4.65 eggs. This last figure is I 7 per cent. low on the observed value rather than 5.7 per cent. as 
indicated by the former estimate, and the difference is therefore not trivial.

Secondly, as regards Osborne's recommendation as to the use of half sib data, it is of interest to note the influence of non-random

TABLE I

Regression coefficients of $\mathrm{Y}$ on $\mathrm{y}, \overline{\mathrm{F}}$, and $\overline{\mathrm{P}}\left(\mathrm{h}^{2}=0.045\right)$

\begin{tabular}{|c|c|c|c|c|c|c|}
\hline \multirow{2}{*}{$\begin{array}{c}\text { Independent } \\
\text { variable }\end{array}$} & \multicolumn{3}{|c|}{ Sire } & \multicolumn{3}{|c|}{ Dam } \\
\hline & $\begin{array}{l}\text { No. of } \\
\text { individuals }\end{array}$ & Simple & Partial & $\begin{array}{l}\text { No. of } \\
\text { individuals }\end{array}$ & Simple & Partial \\
\hline$y$ & $\ldots$ & $\ldots$ & $\ldots$ & I & 0.045 & 0.039 \\
\hline$\underset{P}{F}$ & $\begin{array}{r}8 \\
36\end{array}$ & $\begin{array}{l}0.155 \\
0.452\end{array}$ & $\begin{array}{l}0.122 \\
0.425\end{array}$ & $\begin{array}{l}7 \\
8\end{array}$ & $\begin{array}{l}0.13^{8} \\
0 \cdot 155\end{array}$ & $\begin{array}{l}0.124 \\
0.140\end{array}$ \\
\hline
\end{tabular}

environmental effects on relative selective advance. Numerical values are given in table 2 for Osborne.s example (in the notation of this paper, $n=5, m=\mathrm{I} 5, \bar{n}=6, h^{2}=0 \cdot 05, u^{2}=0$ ), several values being adopted for $c^{2}$. It is evident that when heritability is low a quite small non-random environmental component may render the use of half

TABLE 2

Relative selective advance

$\left(n=5, m=15, n=6, h^{2}=0 \cdot 05, u^{2}=0\right.$ )

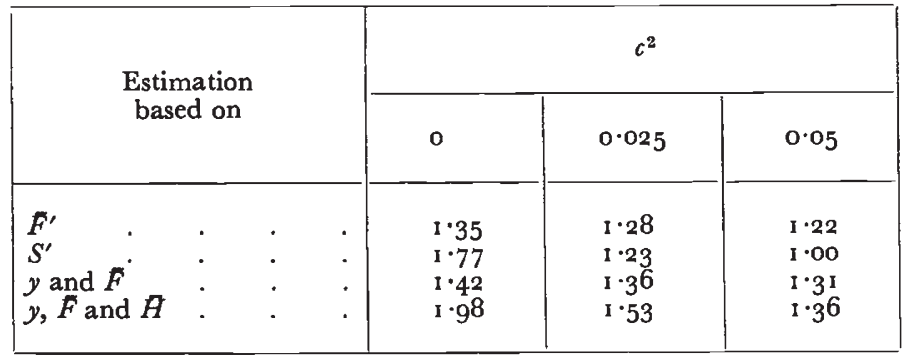

sib data undesirable, and (although Osborne is well aware of this) it is suggested that such data should only be used if positive steps are taken to ensure that such effects are zero and that no a priori assumption of their being so is either adequate or justifiable.

Finally, it may be noted that $k$ and $l$ (which are a measure of the inequality in half sib and progeny family sizes) occur in the denominators of the formulae for the relative selective advance and, being always greater than or equal to I, maximum advance is attained in this latter case when half sib and progeny families are all of equal size. However, as Osborne suggests, inequality in this respect appears to be trivial. 


\section{SUMMARY}

Explicit formulae are presented for the regression coefficients, simple or partial, of the genetic value of an individual on the various mean phenotypic values of its relatives, and for the relative selective advance from the use of those means, these formulae being derived on the assumption that phenotypic value is due to independent additive factors with random and non-random, non-interacting environmental effects. The necessary formulae are also provided for extension to those cases where individuals are inbred, and the estimation of differences in genetic value is discussed for several simple situations.

\section{REFERENCES}

LERnER, I. M. I950. Population Genetics and Animal Improvement. Cambridge University Press.

LERNER, I. M., AND HAZEL, L. N. 1947. Population genetics of a poultry flock under artificial selection. Genetics, 32, 325-339.

Lush, J. L. 1945. Animal Breeding Planș. 3rd Ed. Ames, Iowa : Collegiate Press. LUSH, J. L. 1947. Family merit and individual merit as bases for selection. Amer. Nat., 81, 241-261, 362-379.

MALÉCOt, G. 1948. Les Mathématiques de l'Hérédité. Masson et cie., Paris.

OSBORNE, R. I957. The use of sire and dam family averages in increasing the efficiency of selective breeding under a hierarchical mating system. Heredity, II, 93-I 16. 